\title{
DESEMPENHO DO MODELO SWAT PARA DIFERENTES CRITÉRIOS DE GERAÇÃO DE UNIDADES DE RESPOSTA HIDROLÓGICA
}

\author{
Performance of the swat model using different criteria for generation of bydrologic response units
}

Eduardo de Oliveira Bueno ${ }^{1 *}$; Vinícius Augusto de Oliveira ${ }^{2}$; Marcelo Ribeiro Viola ${ }^{3}$; Carlos Rogério de Mello ${ }^{4}$

\begin{abstract}
${ }^{1}$ Doutorando; Departamento de Engenharia; Universidade Federal de Lavras; eobueno@yahoo.com.br 2 Pesquisador; Departamento de Engenharia Hídrica; Universidade Federal de Pelotas; aovinicius@gmail.com ${ }^{3}$ Professor Adjunto; Departamento de Engenharia; Universidade Federal de Lavras; marcelo.viola@deg.ufla.br ${ }^{4}$ Professor Associado; Departamento de Engenharia; Universidade Federal de Lavras; crmello@deg.ufla.br
\end{abstract}

Artigo enviado em 30/01/2017, aceito em 25/04/2017 e publicado em 07/07/2017.

Resumo - A subjetividade no uso do modelo SWAT, no que diz respeito à discretização espacial da bacia em subbacias juntamente com a geração das unidades de resposta hidrológica (HRUs) foi a questão que motivou a elaboração deste artigo. Foram comparadas as vazões mensais obtidas com o modelo SWAT na simulação de uma bacia hidrográfica com $2030 \mathrm{Km}^{2}$ de área de drenagem (rio Grande, MG), adotando diferentes critérios de geração de HRUs, bem como de discretização espacial em sub-bacias. Baseado nos resultados, conclui-se que a configuração do modelo SWAT com um maior número de sub-bacias e HRUs múltiplas apresentou melhores resultados, na calibração e validação, no passo de tempo mensal, do que o cenário com menor detalhamento da rede de drenagem e apenas uma HRU por sub-bacia. Observou-se também que uma maior discretização da bacia em sub-bacias e HRUs propiciou melhores estatísticas de precisão. Dentre os três critérios de geração de HRUs analisados, houveram poucas diferenças entre os resultados dos métodos que geram uma única HRU por sub-bacia (HRU Predominante e Classes Predominantes). Entretanto, observou-se uma melhoria significativa no desempenho do modelo, expresso nas estatísticas de precisão NS (coeficiente de Nash-Sutcliffe) e PBIAS (tendência), ao se considerar o método que gera mais de uma HRU por sub-bacia (HRUs Múltiplas). Assim, foi possível concluir que o maior grau de discretização espacial proporcionou melhores resultados na simulação hidrológica mensal.

Palavras-Chave - hidrologia, HRUs, simulação hidrológica.

\begin{abstract}
The subjectivity in the use of the SWAT model, regarding the spatial discretization of the basin in subbasins along with the hydrologic response units (HRUs) generation, was the issue that motivated this study. It was compared monthly streamflow obtained by SWAT model simulated to a watershed with $2030 \mathrm{~km}^{2}$ of drainage area (Grande River, MG State), taking into account different criteria for HRUs generation as well as spatial discretization in sub-basins. Based on the results, it was conclude that the configuration of the SWAT model with a higher number of sub-basins and multiple HRUs have produced better results for both calibration and validation procedures, in monthly time step, than the configuration based on both less spatial detailing of the drainage network and only a HRU by sub-basin. It was also observed that a fine discretization of the watershed into sub-basins and HRUs has provided better statistical of precision. Among the three criteria for HRU generation tested in this study, there were observed slight differences between the results of the methods based on a single HRU by sub-basin (Predominant HRU and Predominant Classes). However, it was observed that there was a significant improvement on the model's performance given by the statistics of precision NS (Nash-Sutcliffe coefficient) and PBIAS (bias), when considered the method that generates a greater number of HRU by sub-basin (Multiple HRUs). Thus, it was possible to conclude that the finest spatial discretization has provided better results in monthly hydrologic simulation.
\end{abstract}

Keywords - hydrology, HRUs, hydrology simulation. 


\section{INTRODUÇÃO}

Em simulação hidrológica é usual agregar áreas com comportamento hidrológico semelhante, com o intuito de simplificar a representação de um sistema físico, na expectativa de facilitar a aplicação de modelos, sem deixar de obter resultados confiáveis. No entanto, a principal dificuldade em subdividir bacias hidrográficas em áreas com resposta hidrológica uniforme é definir o que constitui uma área hidrologicamente homogênea. Esta dificuldade depende das características de cada bacia em estudo, com maior ou menor variabilidade, além da experiência do usuário (KOUWEN et al., 1993).

O conceito de Unidades de Resposta Hidrológica (HRUs) é utilizado para a classificação hidrológica de diferentes áreas de uma bacia que apresentem a mesma combinação de declividades, tipos e usos do solo, independentemente da sua localização geográfica (COLLISCHONN et al., 2007). Para Melo Neto et al. (2014), cada HRU representa uma combinação única destes atributos, a fim de facilitar a simulação dos fenômenos físicos que ocorrem dentro dela.

Entre os modelos hidrológicos que adotam o conceito de HRU em sua formulação, destaca-se o SWAT (Soil and Water Assessment Tool), um modelo de base física que permite a análise de diferentes processos em bacias hidrográficas, a partir de parâmetros espacialmente distribuídos (MELO NETO et al., 2014). O SWAT requer informações em três níveis de escala espacial: bacia hidrográfica; sub-bacias e unidades de resposta hidrológica (HRUs). A divisão das sub-bacias em HRUs permite ao modelo simular a evapotranspiração resultante de diferentes combinações de culturas e tipos de solo. O escoamento é calculado para cada HRU e depois propagado para obter os escoamentos nas sub-bacias e, por fim, o deflúvio total na bacia hidrográfica. Desta forma, com a adoção de HRUs, o modelo pode proporcionar uma melhor simulação física do balanço hídrico e aumentar a precisão das estimativas de vazões na bacia em estudo (NEITSCH et al., 2011). Portanto, para a aplicação do modelo SWAT, a definição das HRUs é uma etapa de grande importância, visto que se espera a identificação dos distintos ambientes de comportamento hidrológico homogêneo dentro das sub-bacias, o que é definido pela sobreposição das informações topográficas, pedológicas e de cobertura vegetal. À medida em que se busca uma representação mais precisa da heterogeneidade espacial incorre-se no aumento do número de HRUs. Contudo, do ponto de vista de processamento computacional, um elevado número de HRUs pode significar aumento do tempo e espaço (memória) para processamento. Assim, um equilíbrio entre estes aspectos deve ser observado, sem que haja uma perda na qualidade dos resultados dos modelos (HAVERKAMP et al., 2002).

Os três critérios de geração de HRUs disponíveis no modelo SWAT são: Classe Predominante; HRU Predominante e HRUs Múltiplas. No primeiro e segundo critérios, cada sub-bacia é representada por apenas uma HRU, enquanto no terceiro critério, são geradas múltiplas HRUs para uma mesma sub-bacia. Diversos estudos têm sido desenvolvidos visando investigar os efeitos do critério para definição de HRUs sobre os resultados das simulações com o modelo SWAT, podendo-se destacar HAVERKAMP et al. (2002); MACHADO, VETORAZZI e CRUCIANI (2003); JHA et al. (2004); ARABI et al. (2006); GASMANN et al. (2007); GITHUI e THAYLAKUMARAN (2011) e MELO NETO et al. (2014). Contudo, os resultados são dependentes das escalas espacial e temporal trabalhadas, processos simulados, saídas objeto da modelagem, entre outros. Não existe um consenso na literatura especializada sobre o melhor critério para definição de HRUs a ser adotado no modelo hidrológico SWAT, visando à correta discretização espacial das sub-bacias.

No Brasil, o modelo SWAT tem sido adotado largamente na modelagem de bacias hidrográficas com escalas distintas, para diferentes objetivos: avaliação de impactos de mudanças no uso do solo; potencial de produção e transporte de sedimentos; disponibilidade hídrica; impactos hidrológicos decorrentes de mudanças climáticas, dentre outros (DURÃES, MELLO e NAGHETTINI, 2011; LELIS et al., 2012; PINTO et al., 2013; ANDRADE, MELLO e BESKOW, 2013; PERAZZOLI, PINHEIRO E KAUFMANN, 2013; EDUARDO et al., 2016).

No estado de Minas Gerais, especificamente na região de cabeceira do rio Grande, a qual apresenta importância estratégica para geração hidroelétrica do país, destacam-se alguns estudos de aplicação do modelo SWAT. Andrade, Mello e Beskow (2013) aplicaram o modelo para avaliar o escoamento em uma bacia representativa dos latossolos; Pinto et al. (2013) e Eduardo et al. (2016) para simulação do transporte de sedimentos; Melo Neto et al. (2014) em previsão de vazões considerando o efeito da escala das bacias hidrográficas na simulação dos processos hidrológicos. Nota-se, portanto, diferentes objetivos de aplicação do modelo SWAT na região de cabeceira do rio Grande, entretanto, conforme já destacado sem uma pesquisa básica que norteie o procedimento de geração de HRU.

Neste contexto, objetivou-se neste estudo analisar a influência da metodologia de delimitação de HRUs e de discretização espacial da bacia do rio Grande, 
com seção de controle em Madre de Deus de Minas, em sub-bacias, no desempenho do modelo SWAT para simulação de vazões mensais.

\section{MATERIAL E MÉTODOS}

\section{Região de estudo e banco de dados}

A região em estudo (Figura 1A) situa-se no sul do estado de Minas Gerais, correspondendo à bacia hidrográfica do rio Grande, com seção de controle no posto fluviométrico de Madre de Deus de Minas, totalizando uma área de $2030 \mathrm{Km}^{2}$.

O clima predominante na região de estudo, de acordo com a classificação de Köppen, é "Cwa" na maior parte da bacia, com precipitação média anual em torno de 1600 mm, e "Cwb" junto à Serra da Mantiqueira, onde os índices pluviométricos ultrapassam os $2300 \mathrm{~mm}$. A temperatura média anual é de $18{ }^{\circ} \mathrm{C}$, com mínima média abaixo dos $13{ }^{\circ} \mathrm{C}$ no inverno, e máxima média de $21^{\circ} \mathrm{C}$ no verão (MELLO et al., 2012).

Para aplicação do modelo SWAT, estruturouse um banco de dados hidrometeorológico diário. $\mathrm{Na}$ Figura 1B está apresentada a localização dos postos de monitoramento hidrometeorológico aplicados a este estudo. Os dados de precipitação e vazão foram obtidos do Sistema de Informações Hidrológicas (HidroWeb), da Agência Nacional de Águas (ANA). Para alimentar o gerador climático e o módulo de evapotranspiração do modelo SWAT foram usados dados meteorológicos obtidos do Banco de Dados Meteorológicos para Ensino e Pesquisa (BDMEP) do Instituto Nacional de Meteorologia (INMET).
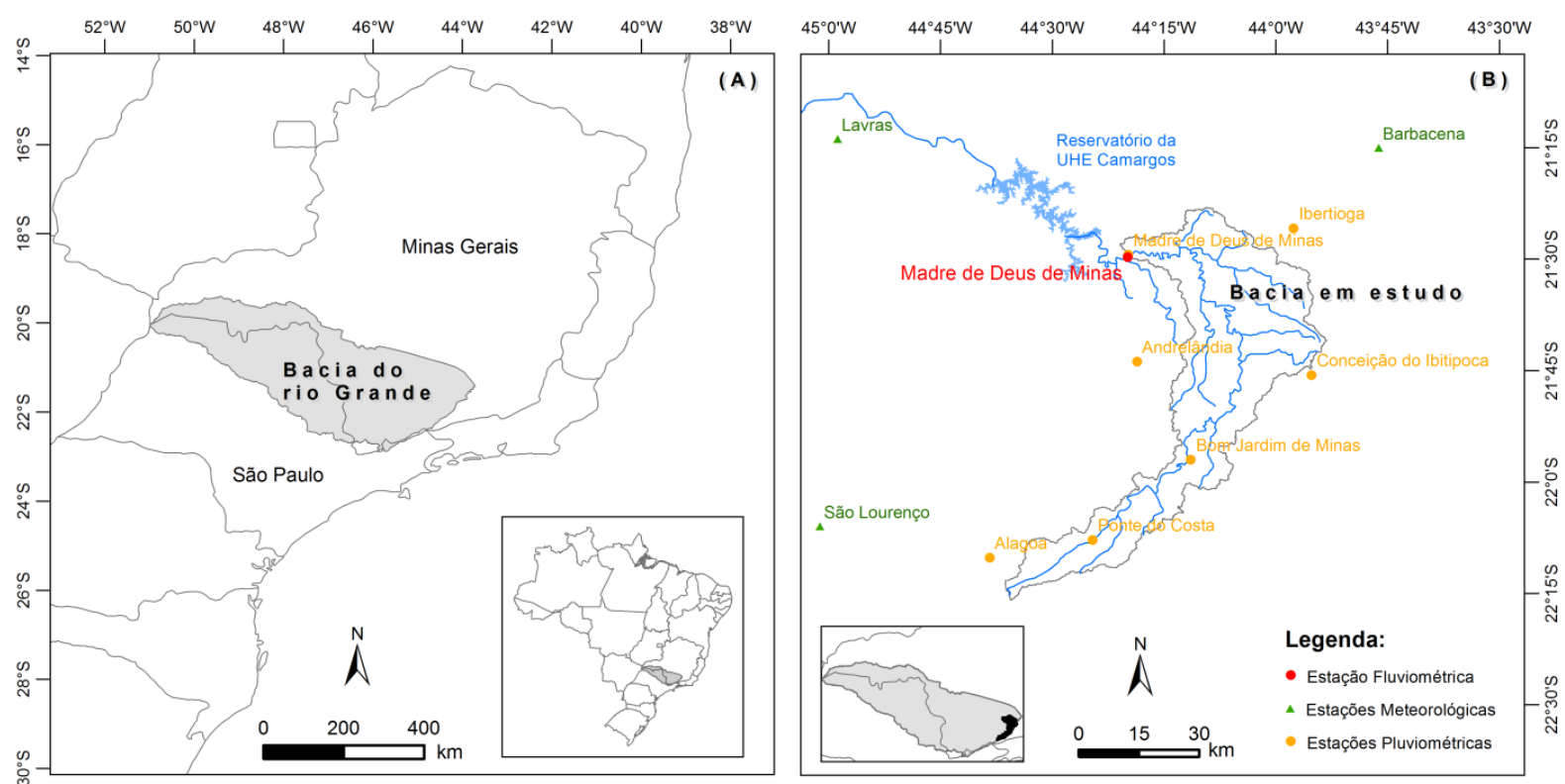

Figura 1 - Localização da bacia hidrográfica do rio Grande no território brasileiro (A), destaque para a bacia em estudo, estações meteorológicas, pluviométricas e fluviométricas utilizadas (B).

A informação base para definição das unidades espaciais no modelo SWAT é o modelo digital de elevação (MDE), tendo sido adotado o MDE ASTER (Advanced Spaceborne Thermal Emission and Reflection Radiometer), com resolução espacial de 30 metros, apresentado na Figura 2a, o qual foi obtido junto ao centro de distribuição da NASA (https://lpdaac.usgs.gov/). As maiores altitudes superam os $2000 \mathrm{~m}$, sendo $2194 \mathrm{~m}$ a altitude máxima, e $913 \mathrm{~m}$ a menor.

Como informações geoespaciais, o modelo ainda requer os mapas de tipos de solo e cobertura e uso. Foi utilizado o mapa de solos disponibilizado pelo Zoneamento Ecológico Econômico do Estado de 
Minas Gerais - ZEE (CURI et al., 2008). A classe de solo predominante é Cambissolo (82,1\%), seguido do Latossolo (11,1\%), Neossolo Litólico (6,6\%) e Argissolo $(0,2 \%)$ (Figura 2c).

A classificação da cobertura vegetal foi gerada a partir de imagens do satélite Landsat 8 (consideradas representativas da condição da bacia durante o período de simulação), utilizando-se o método de classificação supervisionada (Figura 2d). Verifica-se ampla abrangência de pastagem $(70,2 \%$ da área), seguido de floresta mista $(17,2 \%)$, agricultura $(7,8 \%)$, plantio de eucalipto $(3,2 \%)$, campos rupestres $(1,1 \%)$, e outras classes menos expressivas (área urbana, solo exposto, corpos d'água).
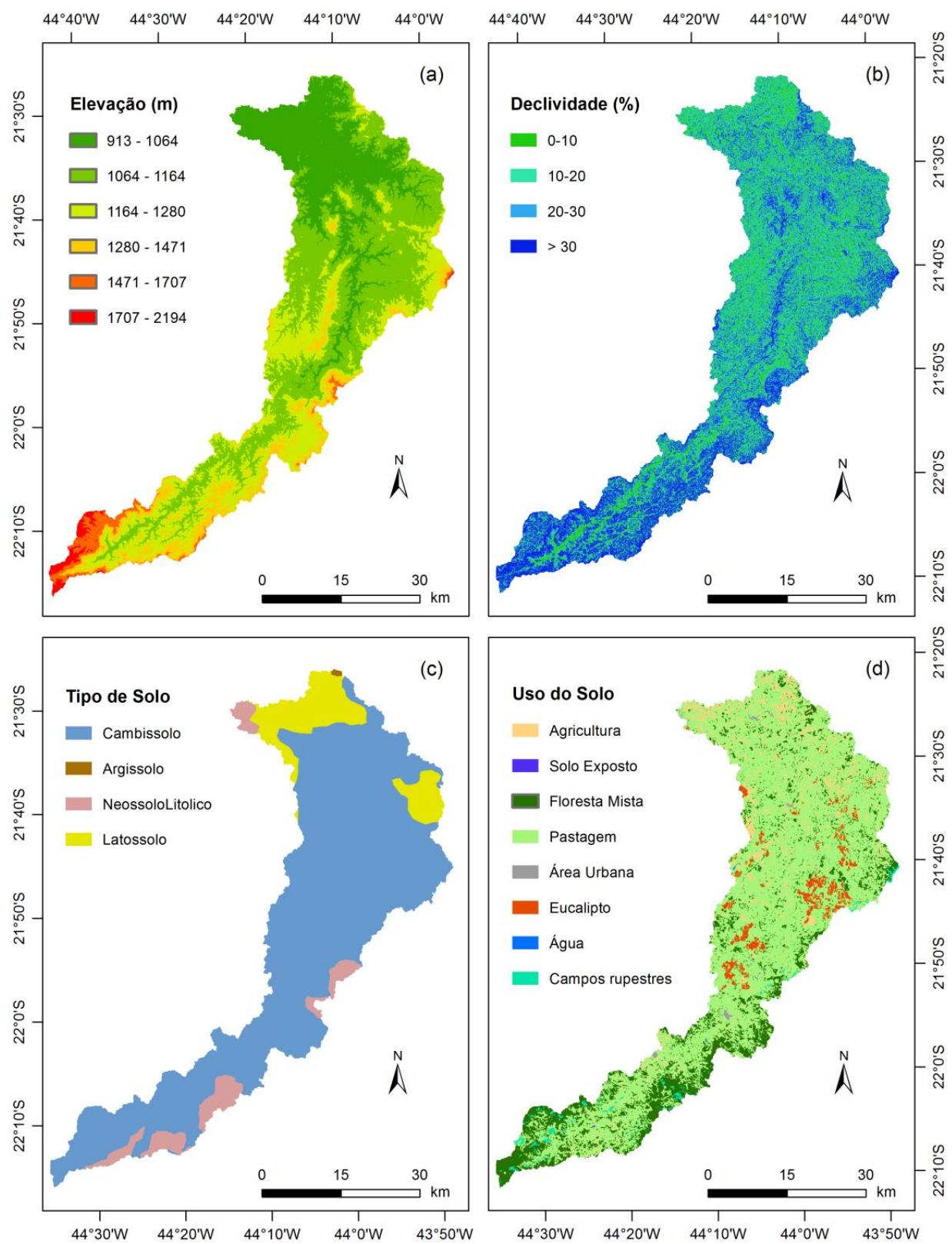

Figura 2 - Modelo digital de elevação (a); mapa de declividades (b); mapa de solos (c) e mapa de uso do solo (d) da bacia do rio Grande com seção de controle no posto fluviométrico de Madre de Deus de Minas. 


\section{Simulação hidrológica}

A simulação hidrológica foi desenvolvida no intervalo mensal em um período de 30 anos, entre 1980 e 2009, sendo selecionados os 15 primeiros anos para a calibração e os demais para validação do modelo hidrológico SWAT. Detalhamentos sobre a estrutura do modelo SWAT podem ser obtidos em NEITSCH et al. (2011) e ABBASPOUR (2015).

O estudo foi desenvolvido na plataforma do programa ArcGIS 10.1 através da extensão ArcSWAT 2012 versão 10_1.15 (WINCHELL et al., 2013). A calibração e validação do modelo SWAT foi feita no programa SWAT-CUP 2012 versão 5.1.6, adotando o algoritmo de calibração SUFI2.

Como no início da simulação ocorrem incertezas associadas ao desconhecimento das condições hidrológicas iniciais, especialmente a umidade inicial do solo, justifica-se a introdução de um período para aquecimento ("warm up") (MELLO et al., 2008). Nesse sentido, os anos de 1980 a 1984 e de 1995 a 1999 foram reservados para aquecimento nas etapas de calibração e validação, respectivamente. Assim, os resultados foram avaliados para os 10 anos subsequentes: 1985 a 1994 (Calibração) e 2000 a 2009 (Validação).

$\mathrm{Na}$ Tabela 1 estão apresentados os 14 parâmetros calibrados na simulação mensal e as faixas de valores admitidas para os operadores durante o processo iterativo, conforme sugerido por Oliveira (2016). A letra no inicio do nome dos parâmetros indica o tipo de operador adotado pelo SWAT-CUP durante o processo de calibração, sendo: a (absolute), $r$ (relative) e $\mathrm{v}$ (replace). Assim, se o operador for do tipo "a" (absolute), soma-se um valor (dentro da faixa de variação indicada na Tabela 1) ao valor do parâmetro resultante da melhor simulação da iteração anterior. Enquanto que, se o operador for do tipo "v" (replace), o programa substitui o valor do parâmetro resultante da melhor simulação da iteração anterior por um novo valor (dentro da faixa de variação indicada na Tabela 1 ). Por fim, se o parâmetro calibrável tiver como operador "r" (relative), o SWAT-CUP multiplica o valor do parâmetro resultante da melhor simulação da iteração anterior por um novo valor (dentro da faixa de variação indicada na Tabela 1). A partir dos novos valores dos parâmetros, procede-se uma nova iteração, buscando a maximização das estatísticas Nash-Sutcliffe (NS) e PBIAS.

O processo de calibração foi conduzido conforme recomendado por Eduardo et al. (2016). Visando padronizar o procedimento de calibração, visto que serão analisados diferentes cenários de HRUs, foram realizadas 5 iterações com 500 simulações para cada aplicação do modelo.

Tabela 1 - Parâmetros calibrados e faixa de variação do operador admitida no processo de calibração no programa SWAT-CUP (OLIVEIRA, 2016).

\begin{tabular}{|c|c|c|}
\hline Parâmetro & Descrição & $\begin{array}{l}\text { Faixa do } \\
\text { Operador }\end{array}$ \\
\hline v_ESCO & $\begin{array}{l}\text { Coeficiente de } \\
\text { compensação de } \\
\text { evaporação de água do } \\
\text { solo }\end{array}$ & 0,5 a 0,95 \\
\hline r_CN2 & $\begin{array}{l}\text { Curva Número inicial } \\
\text { para umidade } \\
\text { antecedente II }\end{array}$ & $-0,1$ a 0,1 \\
\hline v_ALPHA_BF & $\begin{array}{l}\text { Coeficiente de recessão } \\
\text { do escoamento de base }\end{array}$ & 0,0 a 1,0 \\
\hline a_GW_DELAY & $\begin{array}{l}\text { Intervalo de tempo para } \\
\text { recarga do aquífero }\end{array}$ & -30 a 60 \\
\hline a_GWQMN & $\begin{array}{l}\text { Limite de água no } \\
\text { aquífero raso para } \\
\text { ocorrência de fluxo de } \\
\text { base }\end{array}$ & -1000 a 1000 \\
\hline v_CANMX & $\begin{array}{l}\text { Quantidade de água } \\
\text { máxima interceptada } \\
\text { pela vegetação }\end{array}$ & 0 a 30 \\
\hline v_CH_K2 & $\begin{array}{l}\text { Condutividade } \\
\text { hidráulica efetiva do } \\
\text { canal }\end{array}$ & 0 a 10 \\
\hline v_CH_N2 & Número de Manning & $-0,01$ a 0,2 \\
\hline v_EPCO & $\begin{array}{l}\text { Coeficiente de absorção } \\
\text { de água pelas plantas }\end{array}$ & 0,01 a 1,0 \\
\hline v_GW_REVAP & $\begin{array}{l}\text { Coeficiente de ascensão } \\
\text { da água à zona saturada }\end{array}$ & 0,02 a 0,2 \\
\hline a_REVAPMN & $\begin{array}{l}\text { Limite de água no solo } \\
\text { para ocorrência da } \\
\text { ascensão capilar }\end{array}$ & -1000 a 1000 \\
\hline r_SOL_AWC & $\begin{array}{l}\text { Capacidade de } \\
\text { armazenamento de água } \\
\text { no solo }\end{array}$ & $-0,05$ a 0,05 \\
\hline r_SOL_K & $\begin{array}{l}\text { Condutividade } \\
\text { hidráulica saturada do } \\
\text { solo }\end{array}$ & $-0,1$ a 0,1 \\
\hline v_SURLAG & $\begin{array}{l}\text { Coeficiente de } \\
\text { retardamento do } \\
\text { escoamento superficial }\end{array}$ & 0,01 a 24 \\
\hline
\end{tabular}

O processo de calibração adotado no programa SWAT-CUP foi o automático, buscando-se a maximização da função objetivo selecionada no algoritmo SUFI-2: o coeficiente estatístico NashSutcliffe (NS) (NASH e SUTCLIFFE, 1970). Moriasi et al. (2007) apresentaram a seguinte classificação para o coeficiente NS, considerando simulações mensais: 0,75 
$<$ NS $>1,00$ o modelo é considerado muito bom; 0,65 $<\mathrm{NS}<0,75$ o modelo é considerado bom; entre $0,50 \mathrm{e}$ 0,65 satisfatório; e abaixo de 0,50 inadequado.

Além do coeficiente NS foi calculada a estatística PBIAS, que mede a tendência média (em percentual) dos valores simulados serem maiores ou menores do que os dados observados, sendo o seu valor ideal igual a 0 . Valores positivos indicam que a simulação subestimou os valores observados, enquanto valores negativos sugerem uma superestimativa. Van Liew et al. (2007) apresentam a seguinte classificação para este coeficiente em aplicações do modelo SWAT: |PBIAS | $<10 \%$, muito bom; $10 \%<\mid$ PBIAS $\mid<15 \%$, bom; $15 \%$ $<\mid$ PBIAS $\mid<25 \%$, satisfatório; | PBIAS $\mid>25 \%$, o modelo está inadequado.

Apresenta-se a seguir a formulação das estatísticas de precisão utilizadas:

$\mathrm{NS}=1-\frac{\sum_{1}^{n}\left(Q_{o}-Q_{c}\right)^{2}}{\sum_{1}^{n}\left(Q_{o}-\overline{Q_{o}}\right)^{2}}$

PBIAS $=100\left[\frac{\sum_{1}^{n}\left(Q_{o}-Q_{c}\right)}{\sum_{1}^{n} Q_{o}}\right]$

Em que, $Q_{o}$ é a vazão observada, $\overline{Q_{o}}$ é a vazão média observada e $Q_{c}$ é a vazão estimada, $\mathrm{m}^{3} \mathrm{~s}^{-1}$.

\section{Cenários estudados}

No procedimento de delimitação das subbacias no ArcSWAT é sugerido um valor de área para a etapa de criação da rede de drenagem sobre o MDE. Este valor define a área de drenagem mínima para formação da rede de drenagem e, portanto, é importante no detalhamento da rede de drenagem e no número de sub-bacias. Assim para definição do ponto inicial de um curso d'água, o manual do ArcSWAT recomendada a adoção de uma área de drenagem mínima de $2 \%$ da área total em estudo (WINCHELL et al., 2013). Jha et al. (2004) recomendam de 2 a 5\% da área, para bacias entre 2000 e $18000 \mathrm{~km}^{2}$. Arabi et al. (2006) recomendam 4\% para bacias com área inferior a $10 \mathrm{~km}^{2}$.

As unidades de resposta hidrológica (HRUs), definidas como combinações únicas de vegetação, solos e relevo, são geradas individualmente em cada sub-bacia. A interface do ArcSWAT permite ao usuário especificar o critério a ser utilizado para geração das HRUs, sendo: i) classes predominantes: cada sub-bacia terá apenas uma HRU. As classes de solo, vegetação e relevo desta HRU serão as que predominam na sub-bacia, ou seja, as que apresentam maior percentual de ocupação de sua área; ii) HRU predominante: cada sub-bacia terá apenas uma HRU. Após a sobreposição dos mapas de vegetação, solos e relevo, a composição da HRU será dada pela sobreposição correspondente à combinação predominante, ou seja, aquela cuja área representar o maior percentual da sub-bacia; iii) HRUs múltiplas: pode ser gerada mais de uma HRU por sub-bacia. Nesse caso estabelece-se, para cada mapa, um percentual mínimo de ocupação da área da sub-bacia para que seja definida uma HRU. Para esta opção, ao invés de um percentual da área total, pode ser adotado um valor mínimo de área (em ha), o qual será observado para criação das HRUs.

Assim, foram elaborados diferentes cenários de discretização espacial da bacia em sub-bacias e de definição de HRUs, conforme está apresentado na Tabela 2.

Tabela 2 - Configurações dos cenários de discretização da bacia hidrográfica para simulação com o SWAT.

Cenário $\begin{gathered}\text { Critério para } \\ \text { início da rede } \\ \text { de drenagem }\end{gathered} \quad \begin{gathered}\text { Critério para } \\ \text { geração de HRUs }\end{gathered}$

$\begin{array}{lcc}\text { Cenário 1 } & & \text { Classes Predominantes } \\ \text { Cenário 2 } & \begin{array}{c}2 \% \\ \left(40,6 \mathrm{~km}^{2}\right)\end{array} & \text { HRU Predominante } \\ \text { Cenário 3 } & & \begin{array}{l}\text { HRUs Múltiplas } \\ (20 / 10 / 20 \%)\end{array} \\ \text { Cenário 4 } & & \text { Classes Predominantes } \\ \text { Cenário 5 } & 10 \% & \text { HRU Predominante } \\ \text { Cenário 6 } & \left(203 \mathrm{~km}^{2}\right) & \text { HRUs Múltiplas } \\ & & \text { (20/10/20\%) }\end{array}$

Os três primeiros cenários buscam um maior grau de detalhamento espacial da área de estudo em termos do número de sub-bacias. Observa-se que para estes cenários ocorre variação do critério de geração das HRUs.

Para os cenários de 4 a 6 , busca-se um menor número de sub-bacias, comparado aos três primeiros, avaliando-se nesta situação o efeito da adoção de cada critério de definição de HRU. 
Ressalta-se que os limiares (percentuais) mínimos adotados para geração de HRUs múltiplas nos cenários 3 e 6 são aqueles recomendados pelo ArcSWAT: 20\% (classes de usos do solo), 10\% (tipos de solo), e 20\% (classes de declividades). Além disto, o critério de $2 \%$ da área total como área mínima para início da rede de drenagem também é o recomendado (WINCHELL et al., 2013).

\section{RESULTADOS E DISCUSSÃO}

\section{Calibração}

$\mathrm{Na}$ Tabela 3 estão apresentados os resultados do modelo hidrológico SWAT para o período de calibração, considerando os seis cenários analisados.

Tabela 3. Resultados do modelo SWAT para os seis cenários analisados durante a fase de calibração.

\begin{tabular}{ccccc}
\hline Cenário & $\begin{array}{c}\text { No de } \\
\text { Sub-bacias }\end{array}$ & $\begin{array}{c}\text { No de } \\
\text { HRUs }\end{array}$ & NS & $\begin{array}{c}\text { PBIAS } \\
\mathbf{( \% )}\end{array}$ \\
\hline Cenário 1 & 25 & 25 & 0,85 & 10,5 \\
Cenário 2 & 25 & 25 & 0,85 & 10,0 \\
Cenário 3 & 25 & 127 & 0,87 & 4,9 \\
\hline Cenário 4 & 5 & 5 & 0,63 & 26,0 \\
Cenário 5 & 5 & 5 & 0,63 & 26,0 \\
Cenário 6 & 5 & 35 & 0,73 & 17,9 \\
\hline
\end{tabular}

De forma geral, os coeficientes estatísticos mostraram desempenho satisfatório do modelo na simulação contínua de vazões mensais do rio Grande no período de calibração. Para todos os cenários estudados, o coeficiente de Nash-Sutcliffe (NS) superou o limite inferior $(0,50)$ da classificação proposta Moriasi et al. (2007), indicando que o modelo é adequado para simulação. Para os cenários 1 a 3 (maior número de subbacias) o coeficiente NS foi superior a 0,75 , classificando o modelo como muito bom. Em termos da estatística PBIAS, os valores positivos indicam uma subestimativa da vazão média observada. Para os cenários 1 a 3 , o modelo continua sendo considerado como muito bom, conforme a classificação sugerida por Van Liew et al. (2007), com melhor desempenho para o cenário 3 (maior número de HRUs). Entretanto, as calibrações para os cenários 4 e 5 resultaram em valores elevados de PBIAS, sendo classificados como inadequados para simulação.
Em uma análise dos critérios de discretização espacial da rede de drenagem, observa-se que as configurações com maior número de sub-bacias (cenários 1 a 3: 25 sub-bacias) apresentaram resultados (NS e PBIAS) de melhor qualidade estatística que os cenários com menor detalhamento da rede de drenagem, ou seja, um menor número de sub-bacias (cenários 4 a 6: 5 sub-bacias). A Figura 3 ilustra, em termos dos hidrogramas observados e estimados, os resultados dos cenários 1, 2 e 3 (Figura 3A) e 4, 5 e 6 (Figura 3B).

Em relação ao critério de geração de HRUs, considerando apenas o coeficiente NS, observa-se que os cenários 1 e 2, com apenas uma HRU por sub-bacia, apresentaram resultados iguais entre si e muito próximos ao cenário 3 (HRUs múltiplas). Esta observação é validada também para os cenários 4 a 6 (de menor discretização espacial em sub-bacias), nos quais, houve um desempenho ligeiramente melhor para o cenário 6 (HRUs múltiplas). Observa-se que a redução da precisão do modelo que adota apenas uma HRU por sub-bacia em comparação ao que apresenta HRUs múltiplas, é maior para os cenários com menor número de subbacias, condizente com os resultados apresentados por Haverkamp et al. (2002) e Machado, Vetorazzi e Cruciani (2003).

Considerando-se a estatística PBIAS, os modelos elaborados com o critério de HRUs Múltiplas (cenários 3 e 6) se sobressaíram em comparação àqueles elaborados com apenas uma HRU por sub-bacia (cenários 1 e 2; 4 e 5), contrariando as conclusões apresentadas por Gassman et al. (2007), que afirmaram que as vazões simuladas pelo modelo SWAT, em diferentes estudos de casos, foram insensíveis aos critérios de geração das HRUs. Githui e Thayalakumaran (2011) identificaram melhor desempenho do modelo SWAT na simulação de vazões mensais para configuração com menor quantidade de HRUs. Ao considerarmos o erro na estimativa da vazão média (ou do volume de escoamento), representado pela estatística PBIAS, foi obtida uma redução de $53 \%$ ao adotar o cenário 3 (HRUs Múltiplas), em relação ao cenário 1 (apenas uma HRU por sub-bacia).

Ainda considerando o critério de geração de HRUs, os resultados indicaram que a adoção dos critérios de geração de apenas uma HRU por sub-bacia (Classes Predominantes ou HRU Predominante) não resultou em diferenças nas estatísticas de precisão adotadas para avaliar a qualidade da simulação. Portanto, a configuração do modelo SWAT que resultou na melhor simulação para o período de calibração foi a do cenário 3 (maior número de sub-bacias e HRUs múltiplas). Em geral, conforme ilustrado na Figura 3A, o ajuste do hidrograma simulado por este cenário ao 
observado foi bom, subestimando alguns períodos de estiagem $(1985,1986,1987)$ e superestimando um pouco alguns picos de cheias (como nos anos de 1991 e 1992).

A permanência das vazões mensais observadas e simuladas no período de calibração está ilustrada na Tabela 4, permitindo avaliar a precisão do modelo na simulação de vazões com diferentes permanências. Observa-se que, em todos os cenários estudados, as vazões estimadas subestimam as vazões observadas, e que os erros obtidos no cenário 3 foram menores que nos demais cenários, corroborando com os resultados anteriores apontados pelas estatísticas NS e PBIAS.

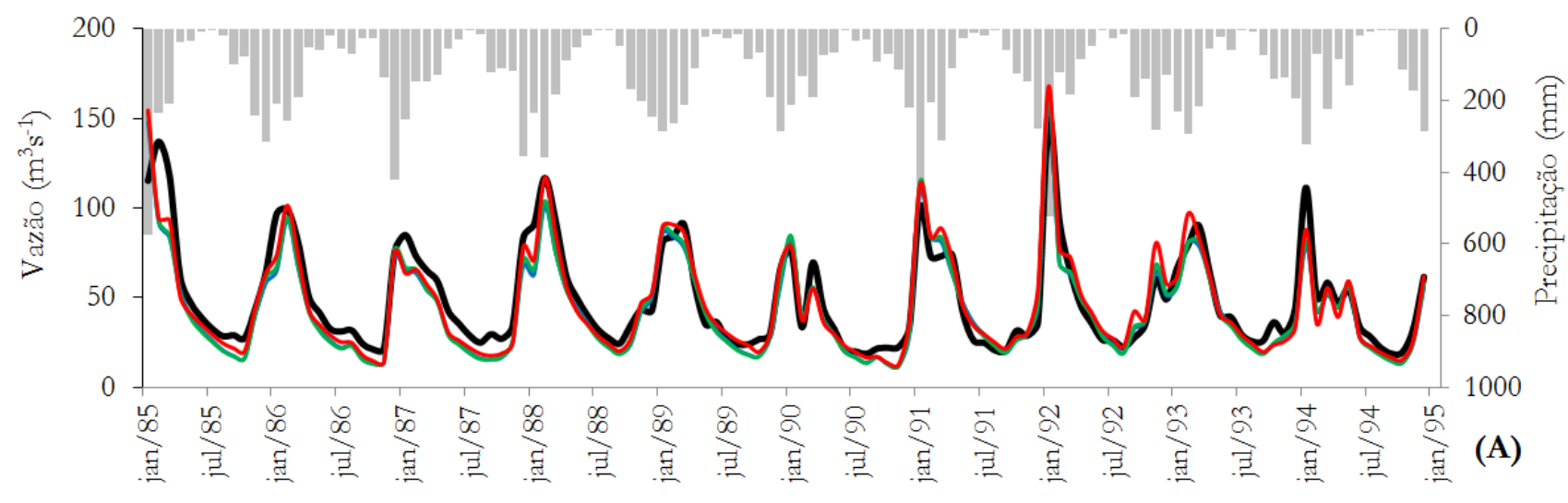

Hietograma

Hidrograma Estimado (Cenário 2)

- Hidrograma Observado Hidrograma Estimado (Cenário 3)

— Hidrograma Estimado (Cenário 1)



Figura 3 - Hidrogramas observado e estimado para a bacia hidrográfica do rio Grande com seção de controle em Madre de Deus de Minas para os cenários 1, 2 e 3 (A) e 4, 5 e 6 (B), no período de calibração. 
Tabela 4. Permanência das vazões mensais do rio Grande observadas e simuladas no período de calibração.

\begin{tabular}{|c|c|c|c|c|c|c|c|c|c|c|c|c|c|}
\hline \multirow[b]{2}{*}{ 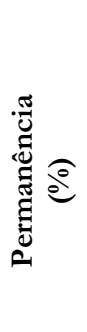 } & \multirow[b]{2}{*}{ 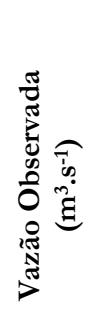 } & \multicolumn{2}{|c|}{ Cenário 1} & \multicolumn{2}{|c|}{ Cenário 2} & \multicolumn{2}{|c|}{ Cenário 3} & \multicolumn{2}{|c|}{ Cenário 4} & \multicolumn{2}{|c|}{ Cenário 5} & \multicolumn{2}{|c|}{ Cenário 6} \\
\hline & & 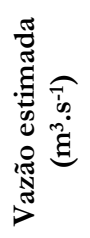 & 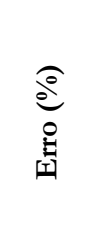 & 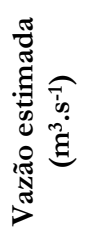 & 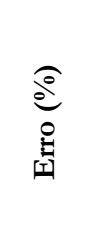 & 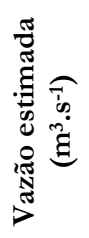 & $\underset{\substack{0 \\
\text { d }}}{\stackrel{0}{a}}$ & 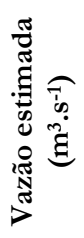 & 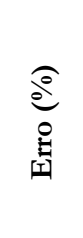 & 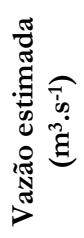 & 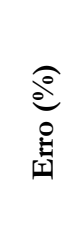 & 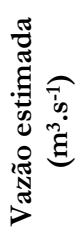 & $\underset{\substack{0 \\
\text { 되 }}}{\stackrel{0}{0}}$ \\
\hline 10 & 90,8 & 80,9 & $-11 \%$ & 82,1 & $-10 \%$ & 86,3 & $-5 \%$ & 74,9 & $-18 \%$ & 74,9 & $-18 \%$ & 77,0 & $-15 \%$ \\
\hline 20 & 73,2 & 64,1 & $-12 \%$ & 66,2 & $-10 \%$ & 71,6 & $-2 \%$ & 51,5 & $-30 \%$ & 51,5 & $-30 \%$ & 58,3 & $-20 \%$ \\
\hline 40 & 44,6 & 44,3 & $-1 \%$ & 43,7 & $-2 \%$ & 44,1 & $-1 \%$ & 33,5 & $-25 \%$ & 33,5 & $-25 \%$ & 39,5 & $-12 \%$ \\
\hline 60 & 32,9 & 29,5 & $-10 \%$ & 29,0 & $-12 \%$ & 30,0 & $-9 \%$ & 22,8 & $-31 \%$ & 22,8 & $-31 \%$ & 26,6 & $-19 \%$ \\
\hline 80 & 26,5 & 20,9 & $-21 \%$ & 20,2 & $-24 \%$ & 23,4 & $-12 \%$ & 17,5 & $-34 \%$ & 17,5 & $-34 \%$ & 18,8 & $-29 \%$ \\
\hline 90 & 22,4 & 17,1 & $-24 \%$ & 17,0 & $-24 \%$ & 19,3 & $-14 \%$ & 15,4 & $-31 \%$ & 15,4 & $-31 \%$ & 14,5 & $-35 \%$ \\
\hline
\end{tabular}

\section{Validação}

A Tabela 5 apresenta os resultados da fase de validação do modelo hidrológico SWAT e a Figura 4 a seguir ilustra os hidrogramas observados e simulados para os seis cenários estudados.

Tabela 5. Resultados do modelo SWAT para os seis cenários analisados durante a fase de validação.

\begin{tabular}{lcccc}
\hline Cenário & $\begin{array}{c}\text { No de } \\
\text { Sub-bacias }\end{array}$ & $\begin{array}{c}\text { No de } \\
\text { HRUs }\end{array}$ & NS & $\begin{array}{c}\text { PBIAS } \\
\mathbf{( \% )}\end{array}$ \\
\hline Cenário 1 & 25 & 25 & 0,89 & 7,0 \\
Cenário 2 & 25 & 25 & 0,89 & 6,3 \\
Cenário 3 & 25 & 127 & 0,89 & 0,4 \\
\hline Cenário 4 & 5 & 5 & 0,81 & 15,0 \\
Cenário 5 & 5 & 5 & 0,81 & 15,0 \\
Cenário 6 & 5 & 35 & 0,87 & 4,3 \\
\hline
\end{tabular}

De uma forma geral, os cenários com maior número de sub-bacias (cenários 1 a 3) apresentaram resultados melhores do que os cenários com menor número (cenários 4 a 6).

Os coeficientes estatísticos mostraram um bom desempenho do modelo na simulação de vazões mensais no rio Grande. Para todos os cenários, o coeficiente de Nash-Sutcliffe (NS) foi superior a 0,75, sendo classificado como muito bom para simulação, conforme classificação proposta Moriasi et al. (2007).

De acordo com os valores de PBIAS obtidos para o período de validação, para os cenários 4 e 5 o modelo é classificado com bom, enquanto que para os demais cenários é considerado como muito bom, conforme a classificação sugerida por Van Liew et al. (2007).

Assim, analogamente à calibração, o melhor desempenho do modelo SWAT foi obtido para o cenário 3 (maior número de sub-bacias e de HRUs).

Nota-se, para o coeficiente NS, que na etapa de validação a diferença de resultados entre os cenários estudados em função do critério de geração de HRUs foi menor do que na fase de calibração. Entretanto, ao analisar a estatística PBIAS nota-se substancial incremento na qualidade do modelo ao adotar um maior número de sub-bacias, passando de 7\% (cenários 1) para $0,4 \%$ (cenário 3); ou mesmo de $15 \%$ (cenário 4) para 4,3\% (cenário 6).

Quanto ao critério de geração de HRUs, se apenas o coeficiente NS for considerado, nota-se que este critério não resultou em diferenças para os modelos com 25 sub-bacias (cenários 1, 2 e 3), e ligeira superioridade para o cenário 6 (HRUs Múltiplas), para o caso de 5 sub-bacias. Entretanto, assim como na calibração, a avaliação da qualidade do ajuste do modelo considerando o indicador PBIAS nos mostra substancial melhora nos resultados adotando HRUs Múltiplas 
(cenários 3 e 6) em relação aos valores obtidos com o critério de apenas uma HRU por sub-bacia (cenários $1 \mathrm{e}$ 2; 4 e 5$)$.

As vazões mensais observadas e simuladas no período de validação, associadas a diferentes permanências, encontram-se na Tabela 6 a seguir. Observa-se que, assim como na calibração, em geral, as vazões estimadas subestimam as vazões observadas, com menores erros novamente associados ao cenário 3. O desempenho do modelo foi pior para simulação das vazões de estiagem, para todos os cenários estudados, em comparação às estimativas das vazões médias e máximas.

Portanto, para a bacia hidrográfica do rio Grande com seção de controle em Madre de Deus de Minas, dentre os cenários estudados para a simulação com o modelo hidrológico SWAT, os melhores resultados foram obtidos pela fixação do valor de $2 \%$ da área total como área mínima para início da rede de drenagem e HRUs múltiplas com os limiares (percentuais) mínimos adotados de 20\% (classes de usos do solo), 10\% (tipos de solo) e $20 \%$ (classes de declividades).

\section{CONCLUSÕES}

Os resultados obtidos demonstraram que o modelo SWAT aplicado à bacia do rio Grande com seção de controle no posto fluviométrico de Madre de Deus de Minas, com área de drenagem de $2030 \mathrm{~km}^{2}$, teve um bom desempenho na simulação mensal, tanto na calibração quanto na validação, de acordo com os valores de referência dos coeficientes NS e PBIAS e pela análise dos erros de vazões de referência extraídas da curva de permanência.

A configuração do modelo SWAT com maior número de sub-bacias e a adoção de HRUs múltiplas apresentou melhores resultados na simulação mensal, nas etapas de calibração e validação, quando comparado aos cenários com menor detalhamento da rede de drenagem e apenas uma HRU por sub-bacia para a bacia estudada.
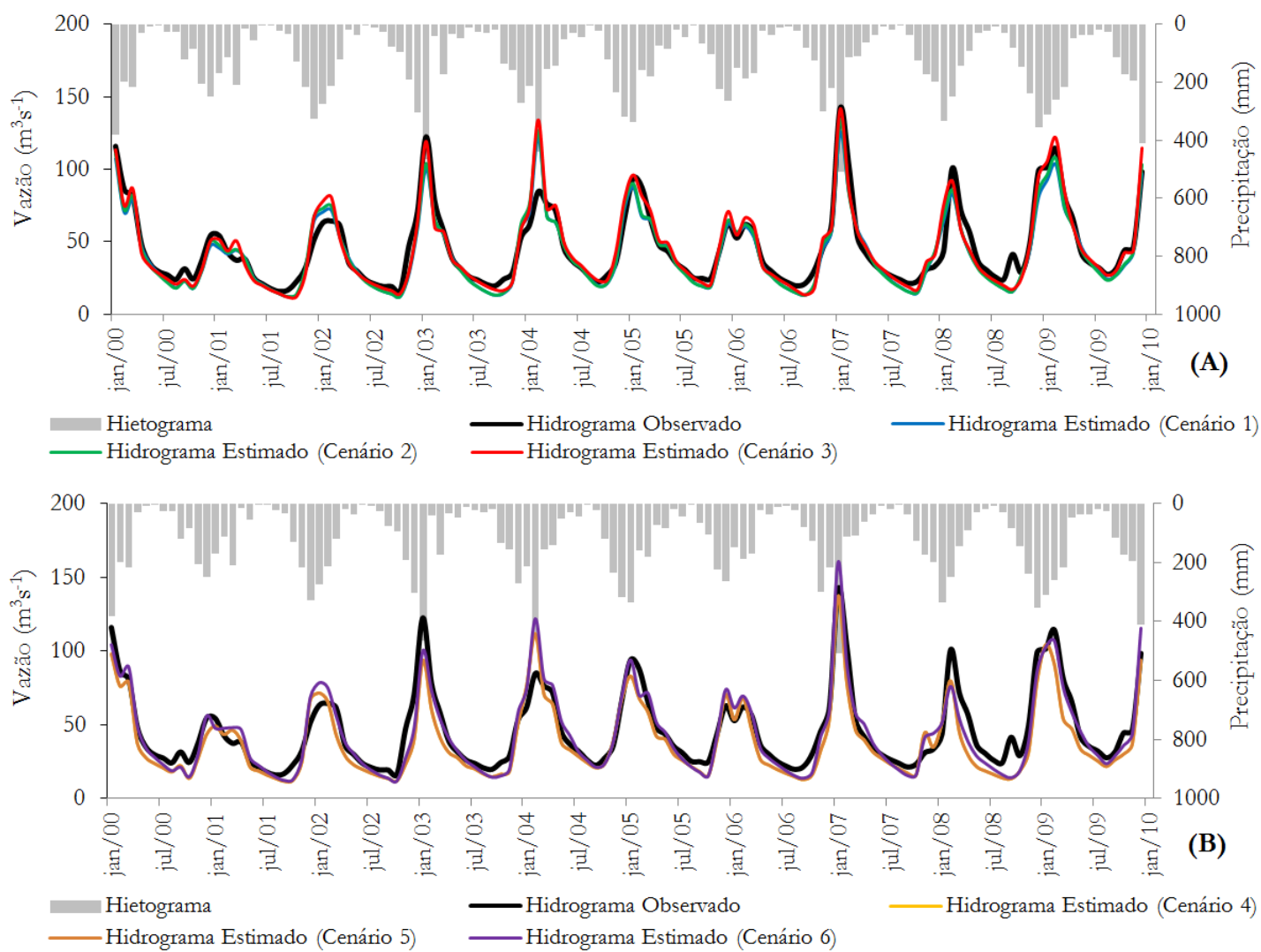

Figura 4 - Hidrogramas observado e estimado para a bacia hidrográfica do rio Grande com seção de controle em Madre de Deus de Minas para os cenários 1, 2 e 3 (A) e 4, 5 e 6 (B), no período de validação. 
Tabela 6. Permanência das vazões mensais do rio Grande observadas e simuladas no período de validação.

\begin{tabular}{|c|c|c|c|c|c|c|c|c|c|c|c|c|c|}
\hline \multirow[b]{2}{*}{ 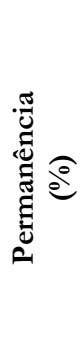 } & \multirow[b]{2}{*}{ 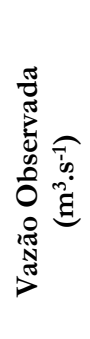 } & \multicolumn{2}{|c|}{ Cenário 1} & \multicolumn{2}{|c|}{ Cenário 2} & \multicolumn{2}{|c|}{ Cenário 3} & \multicolumn{2}{|c|}{ Cenário 4} & \multicolumn{2}{|c|}{ Cenário 5} & \multicolumn{2}{|c|}{ Cenário 6} \\
\hline & & 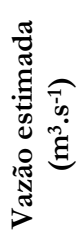 & 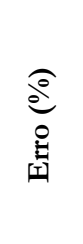 & 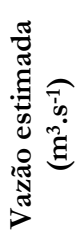 & $\underset{\substack{0 \\
0}}{\stackrel{0}{\varrho}}$ & 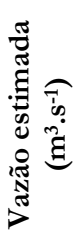 & 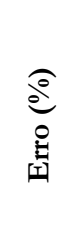 & 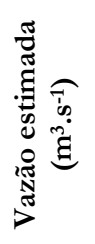 & 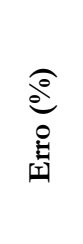 & 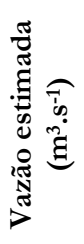 & 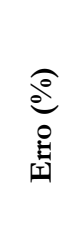 & 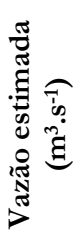 & 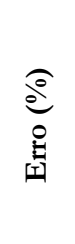 \\
\hline 10 & 84,9 & 73,9 & $-13 \%$ & 77,0 & $-9 \%$ & 85,4 & $1 \%$ & 76,9 & $-9 \%$ & 76,9 & $-9 \%$ & 81,1 & $-4 \%$ \\
\hline 20 & 63,3 & 63,7 & $1 \%$ & 64,8 & $2 \%$ & 68,0 & $7 \%$ & 59,0 & $-7 \%$ & 59,0 & $-7 \%$ & 68,9 & $9 \%$ \\
\hline 40 & 43,5 & 43,6 & $0 \%$ & 43,5 & $0 \%$ & 44,5 & $2 \%$ & 38,2 & $-12 \%$ & 38,2 & $-12 \%$ & 44,2 & $2 \%$ \\
\hline 60 & 31,3 & 29,3 & $-6 \%$ & 28,6 & $-9 \%$ & 30,2 & $-3 \%$ & 25,0 & $-20 \%$ & 25,0 & $-20 \%$ & 28,6 & $-9 \%$ \\
\hline 80 & 24,6 & 19,8 & $-19 \%$ & 19,3 & $-22 \%$ & 21,7 & $-12 \%$ & 18,2 & $-26 \%$ & 18,2 & $-26 \%$ & 19,1 & $-22 \%$ \\
\hline 90 & 21,4 & 15,9 & $-26 \%$ & 15,6 & $-27 \%$ & 17,6 & $-18 \%$ & 15,5 & $-28 \%$ & 15,5 & $-28 \%$ & 15,3 & $-28 \%$ \\
\hline
\end{tabular}

\section{REFERÊNCIAS}

ABBASPOUR, K. C. SWAT-CUP: SWAT Calibration and Uncertainty Programs. A User Manual. Swiss Federal Institute of Aquatic Science and Technology EAWAG. 2015.

ANDRADE, M. A.; MELLO, C. R.; BESKOW, S. Simulação hidrológica em uma bacia hidrográfica representativa dos Latossolos na região Alto Rio Grande, MG. Revista Brasileira de Engenharia Agricola e Ambiental, v. 17, n. 1, p. 69-76. 2013.

ARABI, M.; GOVINDARAJU, R. S.; HANTUSH, M. M.; ENGEL, B. A. Role of watershed subdivision on modeling the effectiveness of best management practices with SWAT. J. American Water Resour. Assoc. 42(2): p. 513-528. 2006.

COLLISCHONN, W; ALLASIA, D; DA SILVA, B. C.; TUCCI. C. E. M. The MGB-IPH model for large-scale rainfall-runoff modelling. Hydrological Sciences Journal, 52:5, 878-895. 2007.

CURI, N.; MARQUES, J. J. G. S. M.; MARQUES, A. F. S. M.; FERNANDES, E. I. Solos, Geologia, Relevo e Mineração. In: Zoneamento ecológico-econômico do Estado de Minas Gerais: componentes geofísico e biótico. Lavras: UFLA, 2008. p. 103-136.
DURÃES, M. F.; MELLO, C. R.; NAGHETTINI, M. Applicability of the SWAT model for hydrologic simulation in Paraopeba river basin, MG. Cerne, v. 17, n. 4, p. 481-488. 2011.

EDUARDO, E. N.; MELLO, C. R.; VIOLA, M. R.; OWENS, P. R.; CURI, N. Hydrological simulation as subside for management of surface water resources at the Mortes River Basin. Ciência e Agrotecnologia, v. 40, n. 4, p. 390-404. 2016.

GASSMAN, P. W.; REYES, M. R.; GREEN, C. H. ARNOLD, J. G. The Soil and Water Assessment Tool: Historical Development, Applications, and Future Research Directions. Transactions of the ASABE, v. 50, n. 4, p. 1211-1250. 2007.

GITHUI, F.; THAYALAKUMARAN, K. The effect of discretization of hidrologic response units on the performance of SWAT model in simulating flow and evapotranspiration. In: 19th International Congress on Modelling and Simulation, Perth, Australia, December. 2011. Anais...

HAVERKAMP, S.; SRINIVASAN, R.; FREDE, H. G.; SANTHI, C. Subwatershed spatial analysis tool: Discretization of a distributed hydrologic model by statistical criteria. J. American Water Resour. Assoc. 38(6): p. 1723-1733. 2002. 
JHA, M. K.; GASSMAN, P. W.; SECCHI, S.; GU, R.; ARNOLD, J. G. Effect of watershed subdivision on SWAT flow, sediment and nutrient predictions. J. American Water Resour. Assoc. 40 (3): p. 811-25. 2004.

KOUWEN, N.; SOULIS, E. D.; PIETRONIRO, A.; DONALD, J.; HARRINGTON, R. A. Grouped response units for distributed hydrologic modeling. J. Water Resour. Plann. Manage. 119(3), 289-305. 1993.

LELIS, T. A.; CALIJURI, M. L.; SANTIAGO, A. F.; LIMA, D. C.; ROCHA, E. O. Análise de sensibilidade e calibração do modelo SWAT aplicado em bacia hidrográfica da região sudeste do Brasil. Revista Brasileira de Ciência do Solo, v. 36, p. 623-634. 2012.

MACHADO, R. E.; VETTORAZZI, C. A.; CRUCIANI, D. E. Simulação de escoamento em uma microbacia hidrográfica utilizando técnicas de modelagem e geoprocessamento. Revista Brasileira de Recursos Hidricos. v. 8. n. 1, p. 147-155. jan/mar. 2003.

MELLO, C. R.; NORTON, L. D.; CURI, N.; YANAGI, S. N. M. Sea surface temperature (SST) and rainfall erosivity in the Upper Grande River Basin, Southeast Brazil. Ciência e Agrotecnologia. 36:53-59. 2012.

MELLO, C. R.; VIOLA, M. R.; NORTON, L. D.; SILVA, A. M.; WEIMAR, F. A. Development and application of a simple hydrologic model simulation for a Brazilian headwater basin. Catena. v. 75, n. 3, p. 235247. 2008.

MELO NETO, J. O.; SILVA, A. M.; MELLO, C. R.; MELLO JUNIOR, A. V. Simulação Hidrológica Escalar com o Modelo SWAT. Revista Brasileira de Recursos Hidricos. v. 19, n. 1, p. 177-188. jan/mar. 2014.

MORIASI, D. N.; ARNOLD, J. G.; LIEW, M. W. Van; BINGER, R. L.; HARMEL, R. D.; VEITH, T. Model evaluation guidelines for systematic quantification of accuracy in watershed simulations. Transactions of the $A S A B E$, 50, 885-900. 2007.

NASH, J. E.; SUTCLIFFE, J. V. River flow forecasting through conceptual models part I: a discussion of principles. Journal of Hydrology, 10, 282-290. 1970.

NEITSCH, S. L.; ARNOLD, J. G.; KINIRY, J. R.; WILLIAMS, J. R. Soil and water assessment tool: theorical documentation version 2009. Texas Water Resources Institute Technical. Report No. 406. Texas A\&M University System. September. 2011.

OLIVEIRA, V. A. Impactos antrópicos na hidrologia e no potencial de geração hidroelétrica no Alto rio Grande - MG. 2016. 128 p. Tese (Doutorado em Recursos Hídricos em Sistemas Agrícolas) - Universidade Federal de Lavras, Lavras, 2016.

PERAZZOLI, M.; PINHEIRO, A.; KAUFMANN, V. Efeitos de cenários de uso do solo sobre o regime hídrico e produção de sedimentos na bacia do ribeirão Concórdia - SC. Revista Árvore, v. 37, n. 5, p. 859-869. 2013.

PINTO, D. B. F.; SILVA, A. M.; BESKOW, S.; MELLO, C. R.; COELHO, G. Application of the Soil and Water Assessment Tool (SWAT) for sediment transport simulation at headwater watershed in Minas Gerais state, Brazil. Transactions of the ASABE, v. 56, p. 697-709. 2013.

VAN LIEW, M. W.; VEITH, T. L.; BOSCH, D. D.; ARNOLD, J. G. Suitability of SWAT for the conservation effects assessment project: a comparison on USDA-ARS watersheds. Journal of Hydrological Engineering, v. 12, n. 2, p. 173-189. 2007.

WINCHELL, M.; SRINIVASAN, R.; DI LUZIO, M.; ARNOLD, J. ArcSWAT Interface for SWAT 2012. User's Guide. Blackland Research and Extension Center. Grassland, Soil and Water Research Laboratory. March. 2013. 